\title{
924 THE SPATIAL HIERARCHY OF PRIMARY AND RECURRENT MEDULLOBLASTOMA TUMOR ECOSYSTEMS
}

${ }^{1}$ Laura Donovan, ${ }^{2}$ Bei Hopkins, ${ }^{1}$ Ben Draper, ${ }^{1}$ Rivani Shah, ${ }^{2}$ Kristin Roman, ${ }^{2}$ Bethany Remeniuk*. ' UCL, London, UK; ${ }^{2}$ Akoya Biosciences, Marlborough, MA, USA

Background Medulloblastoma recurrence occurs in approximately $30 \%$ of patients and is universally fatal, presenting one of the most significant unmet clinical challenges in pediatric oncology. It is now clear that medulloblastomas are complex ecosystems, evolving under selective pressure from their microenvironment and cell of origin. Different tumor-immune cell interactions, including, but not limited to, tumor-infiltrating lymphocytes and various tumor suppressive myeloid cell populations, significantly hamper effective treatment strategies for primary, metastatic, and recurrent tumors. Recurrent medulloblastomas are rarely biopsied making biological material for interrogation scarce. Research has assumed that recurrent and primary medulloblastoma tumors have similar biological composition and therefore will respond to the same therapeutic regimens, however, therapies designed using primary biopsies, but tested in Phase I/II trials on children with recurrent disease, have been largely unsuccessful. We hypothesize that there are select immunosuppressive population differences within primary vs. recurrent tumor microenvironments (TME) that need to be elucidated in order to improve treatment modalities and outcomes in pediatric patients.

Methods Using Akoya's MOTiFTM PD-1/PD-L1 Panel: Auto Melanoma Kit, the staining protocol was adapted for optimal staining performance on human brain tissue. Following this, 24-formalin-fixed, paraffin embedded pediatric medulloblastoma samples (primary and recurrent biopsies from 12 patients) were stained for the following markers on the Leica BOND RX. Multispectral images were acquired using the Vectra Polaris, and five regions of interest selected on each image. An analysis algorithm was developed using inForm tissue analysis software, and samples were batch processed and data exported. Cell counts, densities, and spatial parameters were generated using the R-script package phenoptrReports to produce outputs of the image analysis data.

Results Following spectral unmixing and autofluorescence isolation, no signal crosstalk was observed. The average signal intensity counts for all markers was found to be within the recommended ranges of 10-30, with a coefficient of variation of $\leq 15 \%$, indicating successful and consistent staining of the medulloblastoma samples. Comparison between primary vs. recurrent tissues revealed distinctive spatial differences between immune-tumor cell interactions.

Conclusions We have demonstrated successful adaptation of the MOTiF PD-1/PD-L1 Melanoma panel kit in conjunction with the Phenoptics workflow to support examination of the TME in patient-matched primary and recurrent pediatric medulloblastoma tumor biopsies. Our study provides the first insight into distinctive spatial interactions between primary vs. recurrent tissues, which may improve strategies to comprehend cancer progression, immune surveillance, and ultimately the development of rational, targeted therapeutics based on the differences between the tumor compartments and their immune-microenvironment.

Ethics Approval Ethical approval obtained by Brain UK, ref: 20/008. All participants gave consent to use of their material.

http://dx.doi.org/10.1136/jitc-2021-SITC2021.924 